\title{
Inhomogeneous Model for the Investigation of the Optical Properties of the A-Plane Oriented ZnO Epilayers Grown by Plasma-Assisted Molecular Beam Epitaxy
}

Diouf AA*, Lo B, Ngom BD and Beye AC

Nanoscience Laboratory, Solid Physics and Materials Science Group, Faculty of Science and Technology, Dakar Cheikh Anta Diop University, Dakar-Fann, Senegal

\begin{abstract}
Experimentally the a-plane oriented $\mathrm{ZnO}$ showed three types of excitons $\mathrm{A}, \mathrm{B}$ and $\mathrm{C}$. The theoretical investigation became important to get access inside all excitonic parameters impossible with experimental. Authors used recently the Gaussian distribution by using new parameters to fit perfectly the free excitons. In the present work, one presents another model entitled an inhomogeneous model by taking account another form of Gaussian function, to plot and interpret the excitons $\mathrm{A}, \mathrm{B}$ and $\mathrm{C}$ by using as well the same physical parameters than the theory of the spatial resonance dispersion of Hopfield model. The data found with the inhomogeneous model are almost the same than experimental data found the authors.
\end{abstract}

Keywords: Exciton A, B and C; Inhomogeneous model; Reflectivity spectrum; A plane oriented $\mathrm{ZnO}$

\section{Introduction}

The zinc oxide $(\mathrm{ZnO})$ is a semiconductor II-IV investigated since several years, that is why many physical properties of the $\mathrm{ZnO}$ in large number are already known [1-5]. Now a day, the possibility to increase the thins films and hetero-structures in nanoscale with high quality have attracted the attention of the researchers. With its wide band gap $\left(\mathrm{E}_{\mathrm{g}}=3.37 \mathrm{eV}\right)$ and its larger exciton binding energy $(60 \mathrm{meV})$ several applications have been made such as the ultraviolet (UV)stimulated emission induced by the exciton-exciton scattering at moderate pumping intensity [6], the diluted magnetic semiconductor with the authors [7] which demonstrated theoretically that at room temperature, a $\mathrm{ZnO}$ matrix doped with the transitions metals such as $\mathrm{V}, \mathrm{Cr}, \mathrm{Mn}, \mathrm{Fe}, \mathrm{Co}$, and $\mathrm{Ni}$ present a ferromagnetic behavior as well as several others applications [8-18]. Beside the experimental manipulation, the theoretical aspect played an important role for the investigation of all the excitonic parameters impossible for access with the experimental manipulation. That is why, the authors $[8,9,19,20]$ investigated theoretically and respectively the mixed states of excitons and photons by using the quantum theory of a classical dielectric. The temperature dependent PL of the A free exciton peak energy measured in the case of the perpendicular polarization $(\mathrm{E} \perp \mathrm{c})$ by using the Varshni empirical equation and the theoretical reflectivity of the excitons $\mathrm{A}, \mathrm{B}$ and $\mathrm{C}$ in both polarization parallel and perpendicular by using the Gaussian distribution as well as several others researchers. In this article one presents another model unless the Gaussian distribution used by the authors [9] to investigate the experimental reflectivity observed in the a-plane oriented $\mathrm{ZnO}$ in the polarization, parallel and perpendicular, entitled the inhomogeneous model $[21,22]$ in the framework of a numerical investigation [23-27], to plot the exciton A, B and C. One will compare our results with those obtained in Lo B, Diouf AA [8,9] using other theoretical treatments. In Section 2, Computational Method, one will present the inhomogeneous model which will allow us to determine the theoretical reflectivity of the a-plane oriented $\mathrm{ZnO}$ and the results obtained are well discussed in the section 3 .

\section{Computational Method}

To model the optical properties, one used the inhomogeneous model defined by:
For one oscillator:

$\varepsilon_{r}(E)=\varepsilon_{b}+\int \frac{A_{j}}{\left(x^{2}-E^{2}+i \Gamma E\right)} f\left(x, E_{0}\right) d x(1)$

With

$$
f\left(x, E_{0}\right)=\frac{1}{\sqrt{2 \pi \sigma}} \exp \left\{\frac{-\left(x-E_{0}\right)^{2}}{2 \sigma^{2}}\right\}
$$

For $\mathrm{N}$ oscillator:

$$
\varepsilon_{r}(E)=\varepsilon_{b}+\sum_{j=1}^{N} \int \frac{1}{\sqrt{2 \pi \sigma_{j}}} \frac{A}{\left(x^{2}-E^{2}+\Gamma_{j} E\right)} \exp \left\{\frac{-\left(x-E_{0}\right)^{2}}{2 \sigma_{j}^{2}}\right\} d x
$$

With

$$
A_{j}=\left(\frac{h}{2 \pi} \omega_{p j}\right)^{2}=\alpha_{0, j} \frac{h^{2}}{\pi} \omega_{0, j}=\frac{N e^{2} h^{2}}{4 \pi^{2} m^{*} \varepsilon_{0}}, \omega_{p j}
$$

Being similar to a plasma pulsation, $\alpha_{0, j}$ polarizability of the exciton, $\varepsilon_{b}$ the high-frequency dielectric constant of the material (outside the excitonic resonance), $\Gamma$ the spectral width, $h$ the Planck constant, $\omega_{0}$ is the resonance frequency, $N$ the number of particles, $e_{0}$ the elementary charge electron, $m^{*}$ is the effective mass and $x$ is the resonance energy of excitons.

The inhomogeneous model allows to determine the exciton energy $E_{0, j}$, the oscillator strength $A_{j}$ and the parameters for the widening of the excitonic ray $(h / 2 \pi) . \Gamma_{j}$, it includes in general the influence of the

*Corresponding author: Diouf AA, Nanoscience Laboratory, Solid Physics and Materials Science Group, Faculty of Science and Technology, Dakar Cheikh Anta Diop University, Dakar-Fann, Sénégal, Tel: +221 3382505 30; E-mail: aliounedioufaidara@hotmail.fr

Received April 11, 2018; Accepted April 18, 2018; Published April 25, 2018

Citation: Diouf AA, Lo B, Ngom BD, Beye AC (2018) Inhomogeneous Model for the Investigation of the Optical Properties of the A-Plane Oriented ZnO Epilayers Grown by Plasma-Assisted Molecular Beam Epitaxy. J Laser Opt Photonics 5: 186. doi: 10.4172/2469-410X.1000186

Copyright: $(2018$ Diouf AA, et al. This is an open-access article distributed under the terms of the Creative Commons Attribution License, which permits unrestricted use, distribution, and reproduction in any medium, provided the original author and source are credited. 
temperature and the quality of the material (homogeneity, fluctuations in thickness etc.).

Moreover, the modeling of the optical reflectivity, one can do an investigation of the thermal behavior and turbulent of the system with the spectral widening which is a function of the temperature " $T$ " and the effective masse " $m *=0.59 m 0$ " of the exciton defined by:

$$
\Gamma(T)=\sqrt{\frac{k_{B} \times T}{0.59 \times m_{0}}=\beta . T^{1 / 2}}
$$

With

$$
\beta=\sqrt{\frac{k_{B}}{0.59 \times m_{0}}}(4)
$$

T: Temperature

$\mathrm{m}_{0}$ : Mass of a free electron

$\mathrm{k}_{\mathrm{B}}$ : Constant of Boltzmann.

\section{Results and Discussion}

Experimentally the optical characterization of the a-plane oriented $\mathrm{ZnO}$ shows three types of excitons $\mathrm{A}, \mathrm{B}$ and $\mathrm{C}$, according to the choice of the polarization, parallel or perpendicular. The energy of the three excitons have been found experimentally by authors [8] equal to $\mathrm{E}_{\mathrm{A}}=3.398 \mathrm{eV}\left(\omega_{\mathrm{A}}=0.516 \times 10^{14} \mathrm{~s}^{-1}\right), \mathrm{E}_{\mathrm{B}}=3.410 \mathrm{eV}\left(\omega_{\mathrm{B}}=0.518 \times 10^{14}\right)$ and $\mathrm{E}_{\mathrm{c}}=3.438 \mathrm{eV}\left(\omega_{\mathrm{B}}=0.522 \times 10^{14}\right)$. Diouf et al. [9] investigated theoretically and found almost the same values than the experimental values than Lo et al [8]. by using the Gaussian distribution defined by:

$$
R(\omega)=R_{0}+\alpha \cdot \sum_{i=1}^{n}\left(\frac{A_{i}}{\Gamma \sqrt{\frac{\pi}{2}}}\right) \times \exp -\left(\frac{h^{2}\left(\omega-\omega_{0 i}\right)^{2}}{2 \pi^{2} \Gamma_{i}^{2}}\right)
$$

Where the coefficient $\alpha$ is related to the oscillator strength $\left(4 \pi N e_{0}{ }^{2} / m^{*}\right), \omega_{0}$ is the resonance frequency $\mathrm{e}_{0}=\mathrm{e} / 4 \pi \varepsilon_{0}$ with " $e$ " designated as the elementary charge electron $\mathrm{R}_{0}$ the reflection coefficient $A_{i}$ the spectral area and $\Gamma_{i}$ the spectral width. In the present study one built a program from the inhomogeneous model (1) to investigate the optical properties of the a-plane oriented $\mathrm{ZnO}$. The results obtained by simulation are summarized in the Table 1 compared to the results found experimentally by the authors [8] (Table 2). A comparison of the tables shows that the inhomogeneous model Figure 1a and Figure $2 \mathrm{a}$, is more accurate than the model used by the authors [8] to plot the reflectivity curve of the exciton A B. Otherwise one insists on the fact that the parameters summarized in the (Table 1) are used to model the theoretical reflectivity of the exciton A B. In Table 2 one has the values used by the authors [8] to have their best fit of the free excitons. The method used with the inhomogeneous model allowed furthermore the excitons A B Figure $1 b$, to plot the free exciton $C$ shown in the Figure $2 b$ thing

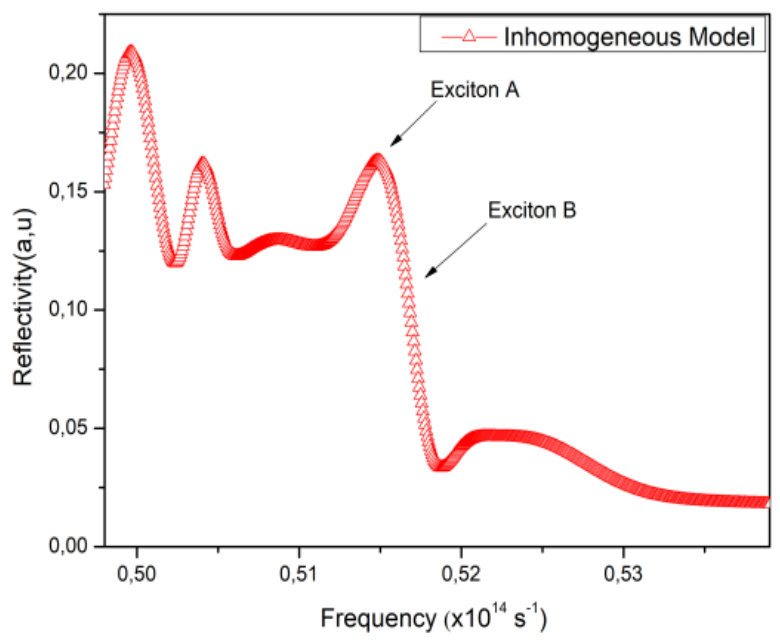

Figure 1a: Theoretical reflectivity of exciton $A$ and $B$.

\begin{tabular}{|c|c|c|c|c|}
\hline Free Exciton & $\begin{array}{c}\text { Frequency }\left(\omega_{0}\right)\left(s^{-1}\right) \\
\text { Energy }(e V)\end{array}$ & Oscillator Strength ( $\alpha$, & Spectral widening $\left(\Gamma,\left(\mathrm{m}^{-\mathrm{s}^{-1}}\right)\right.$ & Effective mass $\left(m^{*}\right)$ \\
\hline A & $\begin{array}{c}5.15 E+13 \\
(E=3.390 \mathrm{eV})\end{array}$ & 1.705 & $\begin{array}{c}3.25 \mathrm{E}+12 \\
(\Gamma=2.14 \mathrm{meV})\end{array}$ & $0.59 \mathrm{~m}_{0}$ \\
\hline B & $\begin{array}{c}5.16 \mathrm{E}+13 \\
(\mathrm{E}=3.401 \mathrm{eV})\end{array}$ & 0.759 & $\begin{array}{c}4.00 \mathrm{E}+14 \\
(\Gamma=26.3 \mathrm{meV})\end{array}$ & $0.59 \mathrm{~m}_{0}$ \\
\hline C & $\begin{array}{c}5.21 \mathrm{E}+13 \\
(\mathrm{E}=3.430 \mathrm{eV})\end{array}$ & -2.044 & $\begin{array}{c}2.53 \mathrm{E}+10 \\
(\Gamma=1.66 \mathrm{meV})\end{array}$ & $0.59 \mathrm{~m}_{0}$ \\
\hline
\end{tabular}

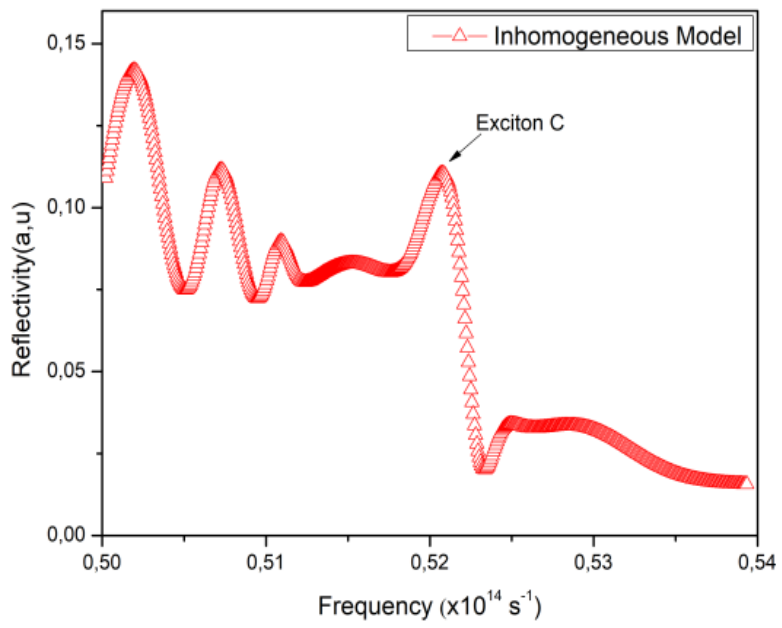

Figure 1b: Theoretical reflectivity of exciton C.

\begin{tabular}{|c|c|c|c|c|}
\hline Free Exciton & Energy $(\mathrm{E})(\mathrm{eV})$ & Oscillator Strength $(\alpha)$ & Spectral widening $(\Gamma)(\mathrm{meV})$ & Effective mass $\left(\mathrm{m}^{\star}\right)$ \\
\hline A & 3.393 & 1.708 & 10.38 & $0.59 \mathrm{~m}_{0}$ \\
\hline B & 3.403 & 0.77 & 11.479 & $0.59 \mathrm{~m}_{0}$ \\
\hline
\end{tabular}

Table 1: Values of A, B and C free exciton frequency $\left(\omega,\left(s^{-1}\right)\right.$, the spatial widening $(\Gamma)$, the effective mass $\left(m^{*}\right)$ and the Area $(A)$ of the reflective curves obtained by using the Gaussian distribution.

Table 2: Values of A and B free exciton Energy $E(e V)$, Oscillator Strength $(\alpha)$, the spatial widening $(\Gamma)$ and the effective mass $\left(m^{*}\right)$ of the exciton obtained by using the Hopfield model [8]. 


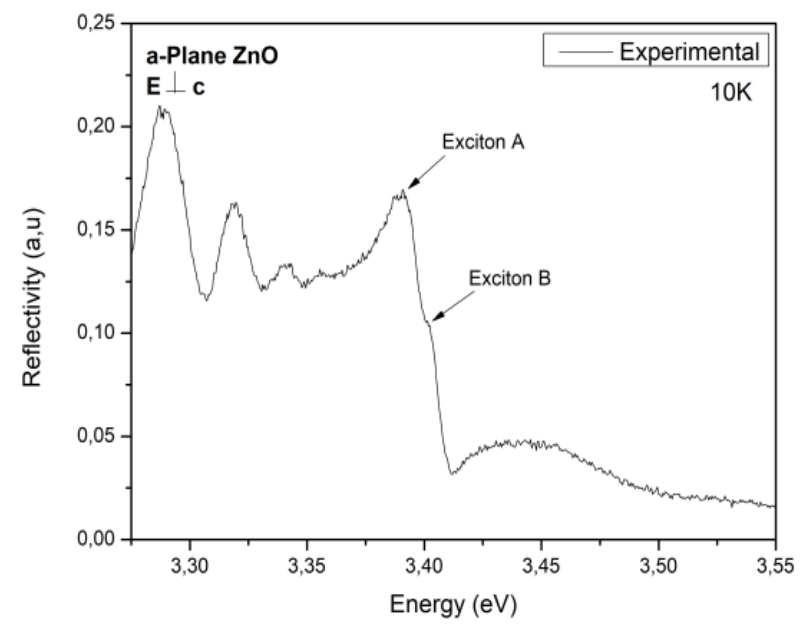

Figure 2a: Experimental reflectivity of exciton $A$ and $B$.

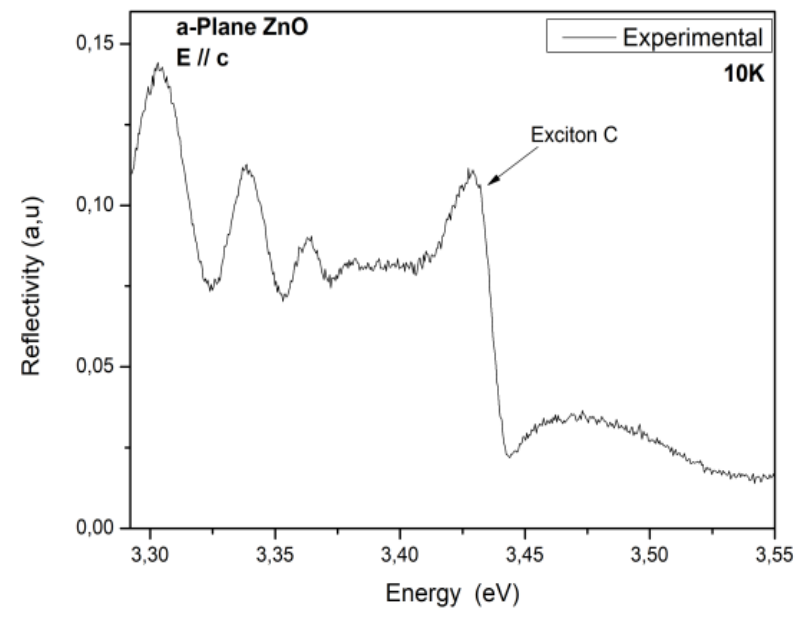

Figure 2b: Experimental reflectivity of exciton C.

found by Diouf [9]. One can explain the phenomenon but the fact that the reflectivity curves are composed by several Gaussian shapes thus with the inhomogeneous model according to the Gaussian distribution it was easy to find the reflectivity curves by playing in the value of the oscillator strength for more accurate. As well a comparison between the authors [8] with the Hopfield model and the inhomogeneous model show important difference summarized in the tables unless the simple Gaussian model (5, used by the authors [9]. One noted that in the range of energy between $3.375 \mathrm{eV}$ and $3.425 \mathrm{eV}$ which represents the reflectivity pic energy range all the models are accurate with the experimental reflectivity curves. But from $3.275 \mathrm{eV}$ to 3.375 $\mathrm{eV}$ representing the range of transparency zone one observed that the inhomogeneous model used plots the whole reflectivity curve obtained experimentally by the authors [8]. One can explain this result by the fact that the inhomogeneous model takes accounts the optical properties when the frequencies $\left(\omega\right.$, are lower than the resonance frequency $\left(\omega_{0}\right.$, exactly like the simple Gaussian distribution used by authors [9]. The parameters used to plot the free exciton $\mathrm{C}$ are summarized in Table 1. The inhomogeneous model can determine separately the both types of widening (Figure 3). The homogeneous widening which depends of the temperature noted $\Gamma$ represents the interaction exciton-photon it

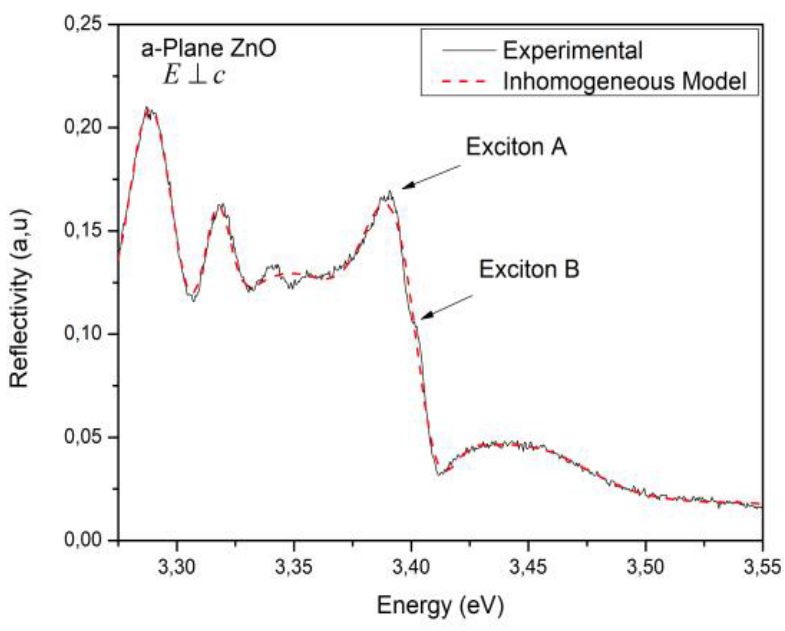

Figure 3a: Set of reflectivity curves of exciton A and B.

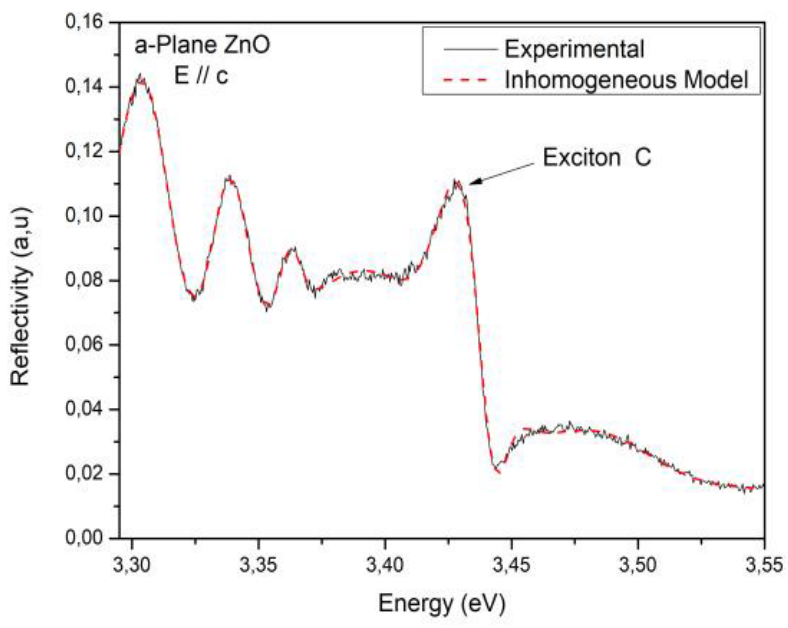

Figure 3b: Set of reflectivity curves of exciton C

increases with the temperature otherwise the inhomogeneous widening depends on the quality of the material through the inhomogeneity of the thickness composition or stress. As well with the inhomogeneous widening it is taken into account through the collection of harmonics oscillators the energy which is distributed according to the Gaussian centered on the average energy $\mathrm{E}_{0}$ and the width halfway up $2 \sigma \sqrt{\ln } 4$.

One used also the spectral widening to investigate the thermal behavior of a-plane oriented $\mathrm{ZnO}$ (Figure 4). One noted that from $8 \mathrm{~K}$ to $300 \mathrm{~K}$ the thermal profile of the sample of $\mathrm{ZnO}$ augments with the enhancement of the temperature. One can explain this phenomenon from the relation (4), by the fact that the temperature is an increase function of the spectral widening. Reason why the authors [8] during their experiments used a cryostat to control the temperature. Unless the others models [28-31] which are limited on the investigation of the c-plane oriented $\mathrm{ZnO}$. But the inhomogeneous model moreover c-plane oriented $\mathrm{ZnO}$ allowed to investigate the structure of the a-plane oriented $\mathrm{ZnO}$ and one plotted in the same time the excitons $\mathrm{A} \mathrm{B}$ and $\mathrm{C}$ something that one did not find in the literature with the others models unless the model presented recently by the authors [32-34]. 


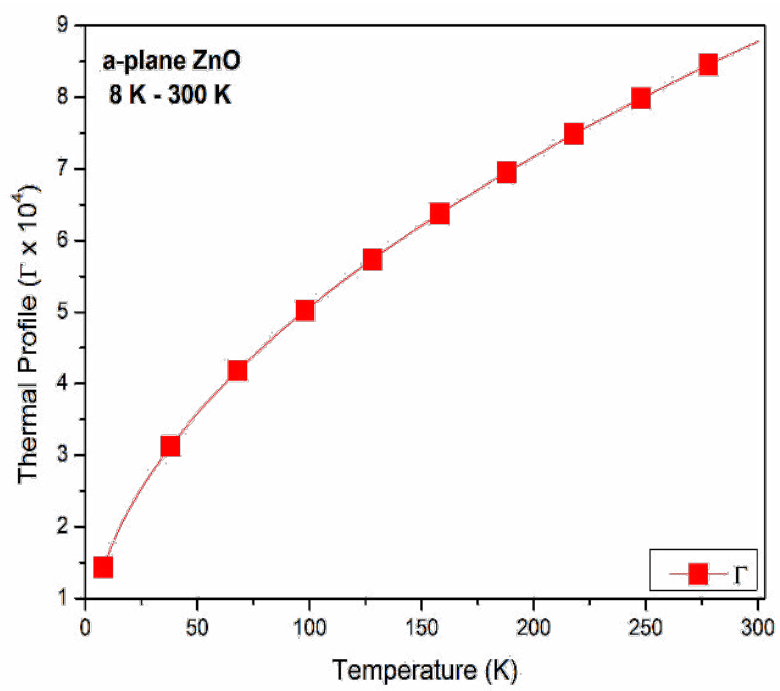

Figure 4: Thermal profile of the a-plane oriented $\mathrm{ZnO}$ from $8 \mathrm{~K}-300 \mathrm{~K}$.

\section{Conclusion}

In this paper one presented another model entitled inhomogeneous model which can be used for the optical characterization. One found the same theoretical reflectivity curves of the free excitons A, B and C in comparison with the experimental results found by Lo et al. by using the same parameters than the authors [8]. Otherwise, one compared the inhomogeneous model with the simple Gaussian distribution used by authors [9], one has the same results the only difference is the spectral area used in the Gaussian distribution [9]. One investigated as well, the thermal profile of the a-plane oriented $\mathrm{ZnO}$ using the spectral widening of the inhomogeneous model. All these results allowed us to present two mathematics models which can be used for the characterization of the nonlinear optical properties with a specific simulation code before getting the experimental results.

\section{Acknowledgment}

Pr. Bassirou Lo gratefully acknowledges about your help for the experimental results for the investigation of the a-plane oriented $\mathrm{ZnO}$. Pr. Beye gratefully acknowledges for your hospitality in your Optoelectronic Laboratory at the University Cheikh Anta Diop of Dakar.

\section{References}

1. Lin B, Fu Z, Jia Y (2001) Green luminescent center in undoped zinc oxide films deposited on silicon substrates. App Phys Lett 79: 943.

2. Béaur L, Bretagnon T, Gil B, Guillet T, Brimont C, et al. (2012) Optical investigations of nonpolar homoepitaxial $\mathrm{ZnO} /(\mathrm{Zn}, \mathrm{Mg}) \mathrm{O}$ quantum wells. Phys Status Solidi C 9: 1320-1324.

3. Subramanyam TK, Srinivasulu BN, Uthanna S (2000) Physical Properties of Zinc Oxide Films Prepared by dc Reactive Magnetron Sputtering at Different Sputtering Pressures. Cryst Res Technol 35: 1193.

4. Rebien M, Henrion W, Bär M, Fischer Ch.-H (2002) Optical properties of ZnO thin films: Ion layer gas reaction compared to sputter deposition. App Phys Lett 80: 3518.

5. Ng-Cheng-Chin F, Roslin M, Gu ZH, Fahidy TZ (1998) On the transmittance properties of electrolytically deposited thin zinc oxide films. J Phys D Appl Phy 31: 71.

6. Li W, Mao D, Zhang F, Wang X, Liu X, et al. (2000) Nucl Instrum Methods Ph Res B169: 59.

7. Sato K, Yoshida HK (2000) Material Design for Transparent Ferromagnets with ZnO-Based Magnetic Semiconductors. Jpn J Appl Phys 39: L555.

8. Lo B, Gaye MB, Dioum A, Mohrain CM, Tall MS (2014) Optical spectroscopy of a-plane-oriented $\mathrm{ZnO}$ epilayers grown by plasma-assisted molecular beam epitaxy. Appl Phys A 115: 257-261.

9. Diouf AA, Lo B, Sambou A, Sakho O, Beye AC (2017) Investigation of the Reflectivity Spectrum of the a-Plane Oriented ZnO Epilayers Grown by PlasmaAssisted Molecular Beam Epitaxy from the Gaussian Distribution. Am Opt Photonics News 5: 50-54.

10. Kunat M, Gil Girol ST, Becker TH, Burghaus U, Wöll CH (2002) Stability of the polar surfaces of $\mathrm{ZnO}$ : A reinvestigation using $\mathrm{He}$-atom scattering. Phys $\mathrm{Rev}$ B 66: 081402R.

11. Xiaodong Y, Jingwen Z, Zhen B, Yongning H, Qing'an X (2005) Glancing incidence $X$-ray analysis of $\mathrm{ZnO}$ thin films and $\mathrm{ZnO} / \mathrm{ZnMgO}$ heterostructures grown by laser-MBE. J Cryst Growth 284: 123-128.

12. Collins RJ, Kleinman DA (1959) Infrared reflectivity of zinc oxide. J Phys Chem Solids, Pergamon Press 11: 190-194.

13. Hengehold RL, Almassy RJ (1970) Electron Energy-Loss and UltravioletReflectivity Spectra of Crystalline ZnO. Phys Rev B 1: 12.

14. Chu S, Olmedo M, Yang Z, Kong J, Liu J (2008) Electrically pumped ultraviolet $\mathrm{ZnO}$ diode lasers on Si. Appl Phys Lett 93: 181106.

15. Lorentz M, Hochmuch M, Schmidt-Grund R, Kaidashevi EM, Grundmann M (2004) Advances of pulsed laser deposition of $\mathrm{ZnO}$ thin films. Ann Phys 13: 59-60.

16. Klucker R, Nelkowski H, Park YS, Skibowski M, Wagner TS (1971) Optical anisotropy of $\mathrm{ZnO}$ in the ultraviolet region. Phys Stat Sol 45: 265.

17. Wu R, Xie C (2004) Formation of Tetrapod ZnO Nanowhiskers and Its Optical Properties. Mater Res Bull 39: 637-645.

18. Venger EF, Melnichuk AV, Melnichuck LYu, Pasechnik A (1995) Surface polariton excitation in ZnO films deposited using ALD. Phys Stat Sol 18: 422-427

19. Hopfield JJ (1958) Theory of the Contribution of Excitons to the Complex Dielectric Constant of Crystals. Phys Rev 112: 1555-1567.

20. Hopfield JJ, Thomas DG (1963) Theoretical and Experimental Effects of Spatia Dispersion on the Optical Properties of Crystals. Phys Rev 132: 563-572.

21. Andreani LC, Panzarini G, Kavokin AV, Vladimirova MR (1998) Effect of inhomogeneous broadening on optical properties of excitons in quantum wells. Phys Rev B 57: 4670.

22. Malpuech G, Kavokin A, Panzarini G (1999) Theory of photon Bloch oscillations in photonic crystals. Phys Rev B 60: 16788.

23. Gilli M, këllezi E (2006) An Application of Extreme Value Theory for Measuring Financial Risk. Comput Econ 27: 1-132.

24. Gilli M, Garbely M (1996) Matchings, covers, and Jacobian matrices. Journal of Economic Dynamics and Control 20: 1541-1556.

25. Golub GH, Van Loan CF (1980) An analysis of the total least squares problem J Numer Anal 17: 883-893.

26. Lawson CL, Hanson RJ, Kincaid DR, Krogh FT (1979) Basic Linear Algebra Subprograms for Fortran Usage. ACM Transactions on Mathematical Software 5: 308-323.

27. Holland TJB (1990) W. H. Press, B. P. Flannery, S. A. Teukolsky \& W. T. Vetterling 1990. Numerical Recipes in Pascal. The Art of Scientific Computing Revised edition. xxii + 759 pp. Cambridge, New York, Port Chester, Melbourne Sydney 127: 376-377.

28. Makino T, Segawa Y, Kawasaki M, Ohtomo A (2006) Analysis on reflection spectra in strained $\mathrm{ZnO}$ thin films. J Cryst Growth 287: 124-127.

29. Reynolds DC, Look DC, Jogai B (1999) Valence-band ordering in ZnO. Phys Rev B 60: 2340

30. Pekar SI (1958) Dispersion of Light in the Exciton Absorption Region of Crystals J Exptl Theoret Phys (U.S.S.R) 34: 1176-1188.

31. Pekar SI (1960) Identification of Excitons with Light Waves in a Crystal and the Macroscopic Theory of Excitons with and without Account of Retardation. J Exptl Theoret Phys (U.S.S.R) 11: 1786-1797.

32. Frenkel J (1930) On Pre-Breakdown Phenomena in Insulators and Electronic Semi-Conductors. Phys Rev 54: 17-44.

33. Wannier GH (1937) The Structure of Electronic Excitation Levels in Insulating Crystals. Phys Rev 37: 191-197. 